\title{
The Influence of Cooperating Self-Driving Car to Highway
}

\author{
Shuo-Yang XU ${ }^{1, \text { a }}$, Lu CHENG ${ }^{1}$, Shi-Yuan QIANG ${ }^{1}$ \\ ${ }^{1}$ Beijing Forestry University, Beijing100083, China \\ a864720564@qq.com \\ *Corresponding author
}

\begin{abstract}
Key words: Traffic congestion, Cooperating self-driving cars, VISSIM, Cellular automata theory.
\end{abstract}
\begin{abstract}
Based on the solution to the problem of highway congestion in Washington state, combined with the theory of cellular automata, the lane changing and longitudinal advance are transformed into mathematical problems, this paper establishes a model of the impact of the Cooperating self-driving cars to the highway by using MATLAB software.
\end{abstract}

\section{Introduction}

Restatement of the Problem. In the United States, traffic capacity is constrained by the design capacity of the road network, affected by the number of roads. This problem does not appear to be as severe as it is during general passage times, but at times of peak traffic congestion, the problem lead to too long time delay is very significant, causing inconvenience to people living and working in many areas of the United States.

In recent years, a variety of solutions have been proposed, but a realistic solution to this problem has not been generated. But a solution that has received much concern and attention has been a trend to adapt to the problem, that is, cooperating self-driving cars. Its proposal is considered as an optimal solution to increase the capacity of the highway without increasing the number of lanes or roads. Because it is the first time to put forward the concept of cooperating self-driving cars, so for now, not yet with the existing traffic flow and the interaction between the two to achieve a balanced position. So the problem to be solved is to study the impact of cooperating self-driving cars on highway congestion in Thurston, Pierce, King and Snohomish counties on the four highways at Interstate 5, Interstate 90, Interstate 405, and State Route 520. First of all, judgment with the proportion of cooperating self-driving cars continue to increase, is how to affect the traffic effect of the highway. Whether there is a turning point in high-speed road capacity, making the road capacity gradually to the equilibrium point. Secondly, the influence of the number of lanes, peak value and average flow rate on traffic flow is calculated by the given data, and studies the influence of cooperating self-driving cars on highway to the situation of positive optimization. Finally, the cooperation between cooperating self-driving cars and their interactions with non-self-driving vehicles should be considered in the model established.

Based on the practical problems to be solved, to propose practical solutions is to establish the ultimate goal of the model. Therefore, it is an effective and direct way to solve this problem by studying the influence of the cooperating self-driving cars on some highways in the United States.

Background. In recent years, with the rapid economic development, science and technology are also high-speed development and progress, traffic is also growing. The problem of highway demand often cannot be solved quickly. The traffic problem represented by traffic jam is a difficult problem which plaguing the big cities all over the world, which seriously restricts the healthy development of a city. So the vehicle communication technology and automatic driving technology research has attracted much attention, people continue to pursue breakthroughs in the field. Cooperating self-driving cars in the forefront of the global forefront of science and technology, the future road traffic will show an unprecedented scene.

The popularity of cooperating self-driving cars will mean a significant reduction in investment in transportation infrastructure such as extra-wide lanes, guardrails, deceleration zones, wide shoulders and even stop signs. Cooperating self-driving cars is a kind of automobile that has the 
characteristics of both communication and autopilot. It is guided by an internal computer using an internal or possibly external sensor that can autonomously navigate. It cannot need the presence of the driver, which is conventional cars cannot be achieved. It not only has a very high accuracy of real-time monitoring of the surrounding environment, but also has the ability to accurately control the vehicle, which can be detected early in the security risks, and timely response to this, so as to avoid accidents. Therefore, cooperating self-driving cars with very high security, if put into the use of the highway traffic can greatly solve many traffic congestion problems. It is understood that, Cooperating self-driving cars equipped with adaptive cruise control system, it is now put into use is a very important one can take into account the highway traffic efficiency and safety systems. Such as BMW's Active Cruise Control with Stop \& Go function.[1]Under the control of the system, each vehicle travels at a specific vehicle speed set in advance. When the electronic sensing device detects the front of the vehicle, the system calculates the distance from the vehicle, it will adaptively adjust the speed to ensure that the distance with the vehicle in front remained at a safe range, thus enhancing traffic safety. Since the scanning frequency of the sensing device is very high, it is possible to detect changes in the vehicle distance in a very short period of time and report it to the electronic control system so that the traffic efficiency can be improved. At the same time, from the environmental point of view, the overall traffic emissions and fuel consumption than conventional vehicles are reduced.[2]

The United States has introduced the latest vehicle communication guidelines to guide vehicles must be able to "communicate." Through the installation of vehicle-to-vehicle (vehicle to vehicle) communication technology, in order to achieve the driver and the surrounding drivers of communication. NHTSA said that this would promote a large number of new anti-car accident applications, and thus avoid the occurrence of car accidents, so this rule requires all car manufacturers must be equipped with V2V technology. ${ }^{[3]}$ Therefore, it is necessary to study the influence of Cooperating self-driving cars on the road traffic, in line with the future communication technology and the inevitable trend of autopilot technology development. This paper is a study of the impact of Cooperating self-driving cars on road traffic in the Washington state highway.

\section{Analysis of the Problem}

The actual traffic congestion problem in Washington State is discussed, and the problem is discussed according to the proposed automatic driving cooperative vehicle scheme without increasing the number of lanes or roads.

First of all, according to the requirements of the subject, you can get to the known and used in the prerequisites are:(1)Lane widths are the standard 12 feet. (2)The nominal speed limit for all these roads is 60 miles per hour.(3)In the provided map and Excel spreadsheet data, map provides a number of highway distribution network. According to the meaning of the question, we select the requirements of the four roads were discussed are: Interstates 5,90,and 405,as well as State Route 520.Among them, Mileposts are numbered from south to north, and west to east. Excel spreadsheet data gives the details of the length of each section, the average daily traffic volume, and the number of lanes, these are the key data for our research.(4)To the time unit for a day, on average, $8 \%$ of the daily traffic volume occurs during peak travel hours. This provides the basis for the study of traffic flow in the later period of the peak period.(5)According to the actual life experience and access to the US road traffic rules, and then determine the traffic lights on the highway does not consider the factors on the impact of road traffic.

Secondly, we combine the known conditions and extract the problems we need to discuss in depth. In the key research on the proposed cooperating self-driving cars on the highway traffic impact of the big problem, in turn to complete the discussion of various types of problems.

Combined with the raw data provided by the topic, analysis and processing of data on not to join the cooperating self-driving cars, we study the region's highway capacity to make an analysis. Combining the original data provided by the topic, we analyze the correlation among the important parameters for data not processed by cooperating self-driving cars. Then we study the situation of joining the cooperating self-driving cars. When the proportion of cooperating self-driving cars from 
$10 \%$ to $50 \%$, has been increased to $90 \%$, taking into account the effect of highway is obviously different, and then analyze the different proportion of the degree of congestion on the highway. As the introduction of cooperating self-driving cars, which means the introduction of communication technology and automatic driving technology, the emergence of new technologies will inject new vitality into the running capacity of road traffic. So we should consider the existence of high-speed road to join the proportion of about how much there will be a turning point in high-speed road capacity to promote road capacity to balance the point, so as to solve the problem of traffic congestion. Of course, the cooperation between cooperating self-driving cars and their interactions with non-self-driving vehicles should be considered in the model established. The factors such as the number of lanes, the peak value and the average flow rate are determined, so as to make the model as possible as possible to the state with the best influence on the highway.

General Definitions.(1)Milepost: A marker on the road that measure distance in miles from either the start of the route or a state boundary.(2)Average daily traffic: The average number of cars per day driving on the road.(3)Interstate: A limited access highway, part of a national system.(4)Increasing direction: Northbound for N-S roads, Eastbound for E-W roads.(5)Decreasing direction: Southbound for N-S roads, Westbound for E-W roads.

Table 1 Variables

\begin{tabular}{|c|c|c|c|c|c|}
\hline Vari & Descriptions & Unit & Vari & Descriptions & Unit \\
\hline $\mathrm{q}$ & $\begin{array}{l}\text { the number of vehicles passing } \\
\text { through a given road section in } \\
\text { a unit time }\end{array}$ & Vehicle/h & $\mathrm{K}_{\mathrm{j}}$ & Blocking density & Vehicle/km \\
\hline V & $\begin{array}{l}\text { the speed of the vehicle at a } \\
\text { given time through the } \\
\text { designated section of the road }\end{array}$ & $\mathrm{km} / \mathrm{h}$ & $\mathrm{v}_{\mathrm{i}}$ & $\begin{array}{l}\text { the speed of the } \\
\text { i-th vehicle }\end{array}$ & $\mathrm{km} / \mathrm{h}$ \\
\hline $\mathrm{k}$ & $\begin{array}{l}\text { the number of vehicles per } \\
\text { unit length of a given section } \\
\text { through a road at a given time }\end{array}$ & Vehicle/km & $\mathrm{ve}_{\mathrm{i}}$ & $\begin{array}{l}\text { the expected } \\
\text { speed of the i-th } \\
\text { vehicle }\end{array}$ & $\mathrm{km} / \mathrm{h}$ \\
\hline $\mathrm{q}_{\mathrm{m}}$ & the maximum traffic flow & Vehicle/ $/ h^{-1}$ & $\mathrm{tv}_{\mathrm{i}}$ & target speed & $\mathrm{km} / \mathrm{h}$ \\
\hline $\mathrm{K}_{\mathrm{m}}$ & $\begin{array}{l}\text { the density of the maximum } \\
\text { traffic flow }\end{array}$ & Vehicle/km ${ }^{-1}$ & $\begin{array}{c}\mathrm{AES} \\
\mathrm{R}\end{array}$ & $\begin{array}{l}\text { actual expected } \\
\text { speed ratio }\end{array}$ & \\
\hline $\mathrm{V}_{\mathrm{m}}$ & $\begin{array}{l}\text { the velocity of the maximum } \\
\text { traffic flow }\end{array}$ & $\mathrm{km} / \mathrm{h}-1$ & AOF & $\begin{array}{l}\text { aggregate } \\
\text { objective function }\end{array}$ & \\
\hline $\mathrm{v}_{\mathrm{f}}$ & the nominal speed limit & $\mathrm{km} / \mathrm{h}$ & CL & $\begin{array}{l}\text { change lane count } \\
\text { ratio }\end{array}$ & \\
\hline $\mathrm{L}_{1}$ & the length of a car & $\mathrm{m}$ & $\mathrm{BD}$ & $\begin{array}{l}\text { lane changed } \\
\text { backward } \\
\text { distance }\end{array}$ & \\
\hline $\mathrm{L}_{2}$ & the length of a truck & $\mathrm{m}$ & OT & $\begin{array}{l}\text { beyond the truck } \\
\text { proportion }\end{array}$ & \\
\hline
\end{tabular}

\section{Data Processing and Analysis}

Correlation Test. According to the original data provided by the table, using Excel to merge the data of each link, and then we are weighted average daily traffic volume. Secondly, the SPSS software was used to test the correlation of factors that may affect the road capacity. The data in Table 2 and Table 3 are derived from the analysis of the provided data. As shown in Table 2, the mean and standard deviation of each data are obtained by using SPSS software, as shown in Table 2.And examines in turn the correlation between the Average daily traffic counts and the Start Milepost, the Total number of lanes, and the Total Milepost.

From the results shown in Table 3, you can visually see the average daily traffic counts and start milepost, the total number of lanes in the 0.01 level (bilateral) on a significant correlation. But it is negatively correlated with the total mileage. 
Table 2Descriptive statistics table

\begin{tabular}{|c|c|c|}
\hline Descriptive statistics & Mean & Standard deviation \\
\hline Average daily traffic counts & 139938 & 48596.13 \\
\hline Start Milepost & 98.89 & 75.79 \\
\hline Total number of lanes & 6.36 & 1.448 \\
\hline Total Mile post & 0.82 & 0.63 \\
\hline
\end{tabular}

Table 3Pearson correlation test results table

\begin{tabular}{|c|c|c|c|}
\hline & Start Milepost & Total number of lanes & Total Mile post \\
\hline Average daily traffic counts & $0.393^{* *}$ & $0.518^{* *}$ & -0.054 \\
\hline
\end{tabular}

Data Processing and Analysis. In order to make the research problems become clearer and play a role in simplification. In this paper, we refer to the traffic flow is defined by the standard length of small cars and large vehicles in a single direction on the road and the formation of traffic, its free from external factors such as: traffic lights, parking or give way signs , Which is not subject to external factors such as: traffic lights, parking or give way to the impact of signs.

There is a concept of fluid in physics, so we consider a traffic flow approximation as the number of vehicles per unit length of a car's cross-section by road, usually in units of Vehicle $/ \mathrm{km}$.

Since it is considered that the flow rate, the velocity, and the density are functions as a function of time and place in practical problems. However, in this paper, we discuss traffic conditions at designated roads or intersections during peak periods, and we consider traffic flow to be constant and continuous without time and place. Define the parameters needed in this paper using the physical concepts in physics: flow, velocity, and density to describe the basic characteristics of traffic flow, and then study the selected problem. The road capacity is evaluated according to the maximum allowable traffic flow.

The flow $q$, velocity $v$, and density $k$ have been explained in Section 2.3.2, and we begin to examine the relationship. In physics, the basic function of flow, velocity, and density is

$$
\mathrm{q}=\mathrm{vk}
$$

We know that there is a close relationship between speed and density, when the vehicle density increases, the speed of the road was forced to reduce. In 1935, Greenshields through the statistical analysis of the observed data, proposed a speed and density between a linear model.

$$
v=v_{f}\left(1-k / k_{j}\right)
$$

Where $v_{f}$ is the density of the speed of $k=0$, that is, in theory, the maximum speed achieved. $v_{f}=60 \mathrm{miles} / \mathrm{h}$ is $96.56 \mathrm{~km} / \mathrm{h} . k_{j}$ is the density at speed $v=0$, called the blocking density. The traffic jam density of single lane is $\mathrm{k}_{j}=1000 /\left(L_{c}+L_{s}\right), L_{c}$ for the body length, $L_{s}$ for the braking safety distance, they are all in meters. Assuming that the length of the car body is $3.8 \mathrm{~m}$, the different vehicle speed under the ordinary traffic density corresponds to the different braking safety distance as shown in Appendix Table4.As can be seen from Table 5, when the vehicle speed is $V_{f} / 2$, the brake safety distance from the head spacing is set to $20 \mathrm{~m}^{[4]}$. The linear equation (2) into (1), you can get the relationship between the traffic flow and density expression

$$
q=v_{f} k\left(1-k / k_{j}\right)
$$

It can be seen from the expression (3) that it is a parabola. The flow $q$ increases as the density $k$ increases. When $k$ reached $k_{j} / 2$, that is when half the blocking density is reached, the flow $q$ takes the maximum value. With the continued increase in $k$, traffic flow is gradually reduced. From (1), (2) we can derive the expression of the relationship between the flow $q$ and velocity $v$ 


$$
q=k_{j} v\left(1-v / v_{f}\right)
$$

It is also a parabola. When velocity $v$ reached $v_{f} / 2$, that is $v_{f}=30 \mathrm{miles} / \mathrm{h}=48.28 \mathrm{~km} / \mathrm{h}$. When it reaches half the expected velocity, the flow $q$ takes the maximum value ${ }^{[5]}$.

Theoretically the maximum traffic volume is $q_{\max }=0.25 \times v_{f} \times k_{j} \times \mathrm{n}$ ( $\mathrm{n}$ is the number of lanes). The largest single-lane traffic flow is about 1050 Vehicle $* \mathrm{~h}^{-1} * \mathrm{~km}^{-1}$. The calculation results are shown in Table 5 of Appendix. However, as shown in appendix table 6, we use SPSS to get average flow (vehicle $/ \mathrm{km}$ ) , maximum, minimum, average value, variance, standard deviation for all segments. And then the peak of the problem can be answered, So the maximum value of 142578.1 ( traffic flow $/ \mathrm{km} * \mathrm{~h}$ ) for the peak.

We use SPSS to analyze the starting mileage and average traffic volume As shown in Figure 1, before the introduction of cooperative self-driving cars, the start mile of Highway 5 which is between 130 and 190, especially between 160 and170, the start mile of Highway 405 which is nearby $0,17,24$, the start mile of Highway 90 which is between 5 and 10 and the start mile of Highway which is between 8 and 10 have the maximum average flow. The volume of traffic exceeds the designed capacity of the road networks. From the map the easy-to-jam sections of Highway 5 is the northeast part of the more complex road in the network. The easy-to-jam sections of Highway 405 and 90 are near intersections.
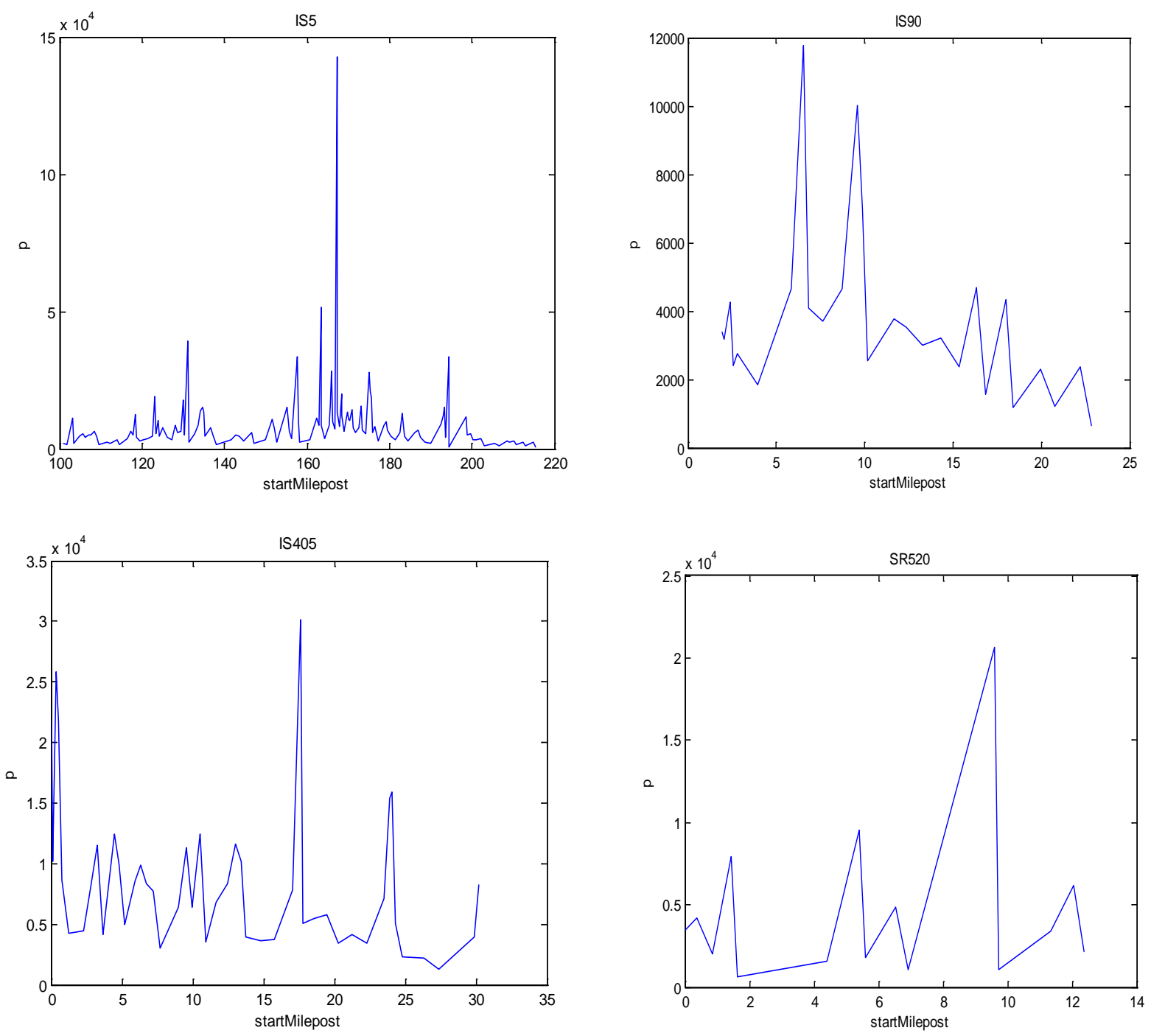

Fig. 1The relationship between start mile and average traffic flow(the unit of p: $\mathrm{pcu}\left[\mathrm{km}^{-1} \mathrm{~h}^{-1}\right.$ ) 


\section{Model Establishment and Solution}

Firstly, this paper uses the above data to simulate traffic through VISSIM traffic simulation software on the road which include co-operating self-driving cars and normal cars. In order to obtain the average travel speeds of each road section. Then the cellular automaton theory is used to simulate the lane changing behavior of the vehicle.

Traffic Flow Efficiency Index.(1)Actual expected speed ratio. In order to evaluate the impact of cooperating self-driving cars on roads, to measure the efficiency of traffic, define the actual expected speed ratio is the radio of average speed and the expected speed,

$$
A E S R=\frac{\sum_{i=1}^{n} \frac{\bar{v}_{i}}{v e_{i}}}{n}=\frac{\bar{v}}{\overline{v e}} .
$$

$v e_{i}$ indicates the expected speed of the i-th car, $\bar{v}_{i}$ indicates the actual average speed of the i-th car; $n$ is the total number of cars; $\bar{v}$ is the total average speed of the road; $\overline{v e}$ indicates the average expected speed of all cars on the road.

In the case of very low car density, all cars are able to drive at the expected speed, $A E S R \rightarrow 1$. In the case of very high car density, the cars drive very slowly due to congestion and $A E S R \rightarrow 0$.

(2)Road traffic capacity. Access to relevant literature, available basic capacity formula:

$$
\mathrm{C}_{\mathrm{b}}=\frac{1000 V_{f}}{\mathrm{~b}+V_{\mathrm{f}} \cdot t / 3.6}
$$

Where $t$ is the reaction time, $b$ is the front and rear car head spacing. Due to the short response time of the cooperating self-driving cars, the required safety distance is also reduced. ${ }^{[6]}$

(3)Maximum road utilization. At the appropriate average speed, the length of all vehicles on the road / length of the road is

$$
C_{c}=\frac{p 1 \cdot n \cdot L 1+p 2 \cdot n \cdot L 2}{L}
$$

Where $p 1$ is the proportion of small cars, $p 2$ is the proportion of large cars. And satisfies $p 2=1-p 1$.

Traffic Flow Safety Index[2].In order to measure the security of traffic flow, we need to define the following indicators

(1)Change Lane Count CL. The increase in the Change Lane Count CL will have a negative impact on road traffic safety, so the average number of lane changes per unit length is measured, Definition: $C L=\frac{N_{C L}}{n \cdot L}$, Where $N_{C L}$ is the total number of lane changes occurring during the simulation, $n$ is the total number of vehicles, $L$ is the length of the road, that is, the number of cells.

(2)Backward distance BD. The lane changing distance is also one of the factors that affect traffic safety when each lane change takes place. Definition: $B D=\frac{\sum_{i=1}^{N_{C L}} d_{i}}{N_{C L}}$, Where $d_{i}$ is the distance between the lane changing vehicle and the rear lane on the target lane when the $i$-th lane change occurs.

(3)Over Take OT. In this model set the cart ratio of $10 \%$, Because the danger of going beyond large vehicles is greater. Definition: $O T=\frac{N_{\text {Отв }}}{N_{\text {От }}}$, Where $N_{\text {От }}$ is the total number of overtaking, $N_{\text {Отв }}$ is the number of times beyond the cart. 
Comprehensive Index of Traffic Flow. Comprehensive traffic flow efficiency index and traffic flow safety index are used to define the traffic flow comprehensive index AOF (Aggregate Objective Function)

$$
A O F=\alpha 1 \cdot A E S R^{\prime}+\alpha 2 \cdot\left(1-C L^{\prime}\right)+\alpha 3 \cdot B D^{\prime}+\alpha 4 \cdot\left(1-O T^{\prime}\right) \text {. Where } \mathrm{Q} \text { is the weight, } \sum_{i=1}^{4} \alpha i=1 \text {, }
$$

$A E S R^{\prime}, C L^{\prime}, B D^{\prime}, O T^{\prime}$ is the variable after normalization. The main purpose of the model is to study the effect of cooperating self-driving cars on the traffic congestion, which is more important. So the weight of traffic flow efficiency index is larger than others. Here we adopt $\alpha 1=0.5, \alpha 2=0.2$, $\alpha 3=0.1, \alpha 4=0.2$. The formula is:

$$
A O F=0.5 \cdot A E S R^{\prime}+0.2 \cdot\left(1-C L^{\prime}\right)+0.1 \cdot B D^{\prime}+0.2 \cdot\left(1-O T^{\prime}\right)
$$

\section{Model: Cooperating Self-Driving Cars (Never Change Lanes)}

Regardless of the lane condition, Cooperating self-driving cars never change lanes. It has the highest security. But obviously the traffic with the efficiency reducing, the average velocity of all vehicles in the formula of traffic flow efficiency will be affected and become smaller. Because the traffic flow safety index only contains the lane change of man-driving cars, the number of changing lanes is reduced and the traffic flow index changes depending on the proportion of the two.

We use VISSIM to simulate the peak period and congested road sections. After inputting the average traffic volume during the peak period $t$, adjusting the parameters of various types of vehicles, and adding the types of cooperating self-driving cars, we get the average velocity of the four freeways $\mathrm{s}$ as shown in appendix table 7.

In order to facilitate the calculation, we suppose the average expected velocity of all vehicles is $80 \mathrm{~km} / \mathrm{h}$, and put it into the formula $A E S R=\frac{\sum_{i=1}^{n} \frac{\bar{v}_{i}}{v e_{i}}}{n}=\frac{\bar{v}}{\overline{v e}}$. Calculate the expected velocity ratio and measure traffic flow efficiency.

Using the MATLAB software to make the image of the expected velocity ratio about the percentage of the Cooperating self-driving cars shown in the following figure 2 :
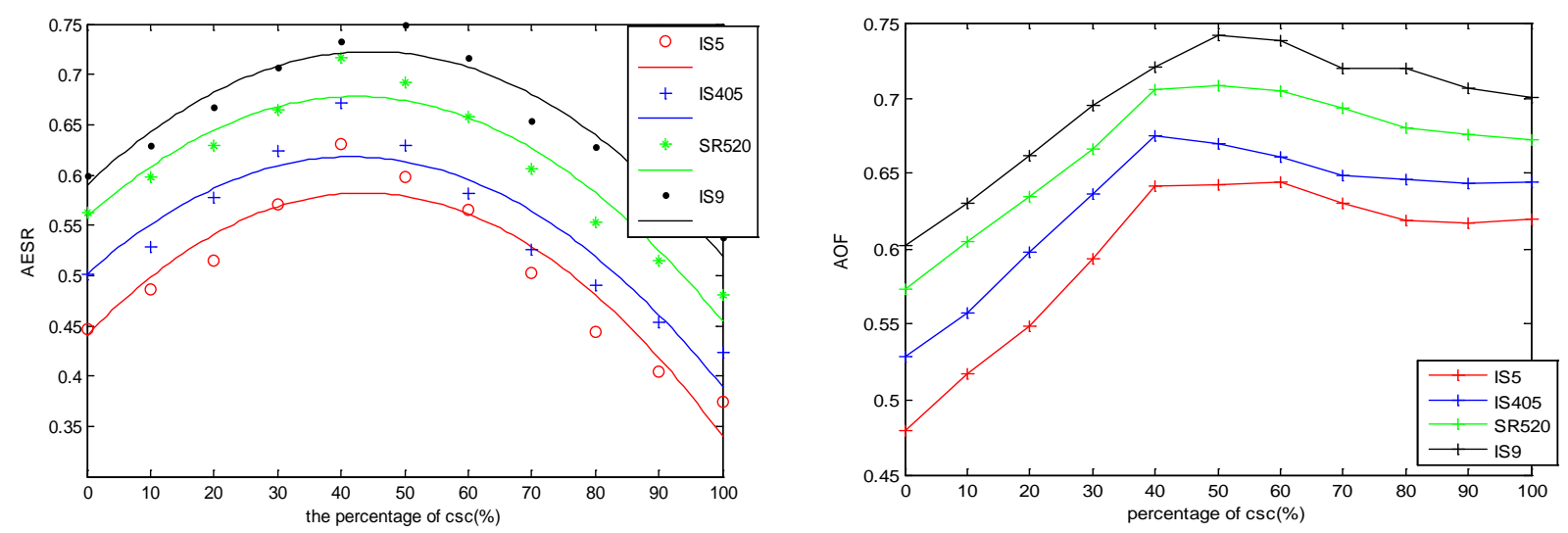

Fig. 2The image of the expected velocity ratio

From figure2 can be seen, the trend of the four highways is roughly the same. When the proportion of Cooperating self-driving cars on the highway gradually increased, the expected velocity ratio AESR increases first and then decreases. The expected velocity ratio reach a maximum when the proportion of Cooperating self-driving cars is about $43 \%$, up to $72 \%$. This is also a turning point. Before this point, the expected velocity ratio will increase continuously. After 
this point, the expected velocity ratio is gradually reduced. The equilibrium point is not existed.

Cooperating self-driving cars does not affect the lane change and driving of normal driving cars, but the traffic flow safety efficiency will be impacted negatively when the Cooperating self-driving car does not change the lane. When the Cooperating self-driving car does not change the lane, use cellular automata model. According to the above-mentioned lane changing criterion, calculate the traffic flow comprehensive index $A O F=0.5 \cdot A E S R^{\prime}+0.2 \cdot\left(1-C L^{\prime}\right)+0.1 \cdot B D^{\prime}+0.2 \cdot\left(1-O T^{\prime}\right)$ by using MATLAB. The result is shown as the following figure6.

From the figure, it can be seen that the integrated traffic flow index first increases and then decreases to a stable value, and the equilibrium point is located at the place where proportion of Cooperating self-driving cars is about $80 \%$. The turning point is about $50 \%$. The more crowded roads such as IS5, the traffic flow comprehensive index is smaller.

\section{Conclusion}

Integrating the discussion and analysis of the problem above, according to requirements of the problem, we believe that when the proportion set is reasonable, the Cooperating self-driving cars have a positive effect on alleviating the congestion of the highway. According to the results under the lane changing model, with the percentage of Cooperating self-driving cars increasing, the traffic flow efficiency decreases first and then increases.

\section{References}

[1]BMW Technology Guide, Active Cruise with Stop \&Go function[M].[S.1.]:[s.n.]. http://www.bmw.com/com/en.

[2] J.J.Hu, Scheduling of connected autonomous vehicle on highway lanes[D], shanghai: Shanghai Jiao tong University School of Electronic Information and Electrical Engineering,2013:1-12.

[3]The United States introduced the latest vehicle communication guidelines, vehicles must be able to "exchange",[EB/OL].Industry news,2016-12-16. http://36kr.com/p/5059487.html.

[4] F.R. Shi, S.W. Yang, J.L. Dai, Discussion on Traffic Capacity of Freeway Section[J]. Journal of Highway and Transportation Research and Development:161-165.

[5] Q.Y. Jiang, J.X. Xie, J.Ye. Mathematical Model. Beijing: Higher Education Press,2015 : 23-49.

[6] W.Zhou,B.G.Wang,Study of the principles and methods for determining LOS and service traffic volume, China Journal of Highway and Transport,Vol.14,No.2,Apr.2001:P90-91. 